\title{
New Guidelines for the Management of Hypertension: Much Controversy, the Good and the Not so Good
}

\author{
Renna NF* \\ Department of Pathology, National University of Cuyo, Argentina
}

Submission: November 27, 2017; Published: December 18, 2017

*Corresponding author: Renna NF, Department of Pathology, National University of Cuyo, Chief of Hypertension Committee of Argentina Federation of Cardiology, Coordinator of Coronary Care Unit, Spanish Hospital of Mendoza, Teacher assistant School of Medicine, UNCuyo, Argentina, Email: nicolasfede@gmail.com

\section{Opinion}

Hypertension is a leading risk factor for morbi-mortality and disability. It is estimated that approximately 875 million adults worldwide have systolic blood pressure above $140 \mathrm{mmHg}$. If the demographic trend is considered and the prevalence of hypertension increases with age, the consequences of hypertension will continue to increase. The 2017 guideline is an update of "The Seven Report of the Joint Committee on Prevention, Detection, Evaluation and Treatment of High Blood Pressure (JNC)" [1,2].

The guidelines provide a comprehensive review for the diagnosis and therapeutics of arterial hypertension. These guides recommend classifying blood pressure into 4 categories: normal (less than 120/80mm Hg); elevated (120-129/lower $80 \mathrm{mmHg})$; stage 1 hypertension $(130-139 / 80 \mathrm{mmHg})$ and stage 2 hypertension (greater 140/greater $90 \mathrm{mmHg}$ ). These categories are designed to facilitate clinical decisions and reflect observational cardiovascular risk data with a gradient of growth as blood pressure increases. You must think that not all populations have the same risk, the cardiovascular risk is not the same in the Mediterranean or in South America than in the USA [1].

An important change is the strong recommendation for taking blood pressure out of the consultation to perform diagnosis and monitoring of blood pressure. Self-measurement (AMPA) and ambulatory monitoring (ABPM) of blood pressure should be encouraged. Evidence IA. This recommendation reflects the frequent inconsistencies between office and home BP values (masked and white-coat hypertension) intensely discussed in these guidelines. In this way, recently, Quer et al. [3] showed fascinating data analysis of home blood pressure metrics. It showed that the BP values significantly decrease after 10 minutes from the initial measurement ( 4.1 and $6.6 \mathrm{mmHg}$ for the diastolic and systolic BP, respectively), and continue to decrease for about 25 minutes $[1,3]$.
Non-pharmacological therapies have high evidence (IA) in the new guidelines for the treatment of hypertension. We must continue to insist with them always. These interventions include weight loss in overweight or obese patients; healthy diet, such as the DASH diet (Dietary Approaches to Stop Hypertension); sodium reduction; the supplement with potassium; the increase of physical activity; and moderation in the consumption of alcoholic beverages. These measures can reduce blood pressure 5 to $10 \mathrm{mmHg}$ [1]

The next point, I find controversial, and that is when we should start pharmacological therapy? and at this point the guidelines is consistent with the changes proposed. Pharmacological therapy is recommended to start it in patients with high cardiovascular risk and blood pressure values of $130 / 80 \mathrm{~mm} \mathrm{Hg}$ or more. Also in those patients without cardiovascular risk or with low risk and blood pressure values above $140 / 90 \mathrm{mmHg}$. The latter does not present changes with respect to the recommendation of the JNC 8, but although the evidence has a strong recommendation, its origin is more indirect, since the data arise from a meta-analysis that does not show significant differences in [1].

To stratify the risk, the guide proposes to use the ASCVD risk score that estimates the risk of myocardial infarction, stroke or death from ischemic heart disease at 10 years [1]. The most recent studies, both randomized and observational trials, reconcile the idea that the lower blood pressure thresholds are beneficial in patients with high cardiovascular risk and those older than 75 years. Undoubtedly, the balance of the potential benefits of controlling the presence of hypertension and the costs of medication, adverse effects and poly-pharmacy for each individual patient should be considered. There is little high quality evidence in the literature about some patient populations, especially the frail elderly [4]. 
I think we can point out that, in these guidelines, the role of self-monitoring has been highlighted, as evidenced by the evidence, and this is very useful for patients to assume a more responsible and active behavior about their disease. However, this new paradigm in the diagnosis of blood pressure, at least for the rest of the world, is still scarce in strong evidence and much anticipated and I do not think it can be reflected in the short term in other consensus or guidelines.

But there are data that stand out in addition to the objectives, such as self-monitoring, the use of fixed doses in severe hypertensive patients, the initiation of early treatment in highrisk cardiovascular patients and in special populations, which are very useful for the management of patients in the doctor's office.

\section{References}

1. Whelton PK, Carey RM, Aronow WS, Casey DE, Collins KJ, et al. (2017) 2017 ACC/AHA/AAPA/ABC/ACPM/AGS/APhA/ASH/ASPC/
NMA/PCNA Guideline for the prevention, detection, evaluation, and management of high blood pressure in adults: A report of the American College of Cardiology/American Heart Association Task Force on Clinical Practice Guidelines. Hypertension doi: 10.1161/ HYP.0000000000000066.

2. Cifu AS, Davis AM (2017) Prevention, detection, evaluation, and management of high blood pressure in adults. JAMA 318(21): 21322134

3. Quer G, Nikzad N, Chief A, Norman A, Vegreville M, et al. (2017) Home monitoring of blood pressure: Short-term changes during Serial Measurements for 56.398 Subjects. A preliminary version presented in American Heart Association Scientific Sessions.

4. Wright JT, Williamson JD, Whelton PK, Snyder JK, Sink KM, et al. (2015) A randomized trial of intensive versus standard blood-pressure control. N Engl J Med 373(22): 2103-2116.

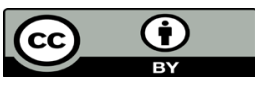

This work is licensed under Creative Commons Attribution 4.0 License DOI: 10.19080/JOCCT.2017.08.555744 